\title{
Stability and Superstability of Ring Homomorphisms on Non-Archimedean Banach Algebras
}

\author{
M. Eshaghi Gordji1, 2,3 and Z. Alizadeh ${ }^{1,2,3}$ \\ ${ }^{1}$ Department of Mathematics, Semnan University, P.O. Box 35195-363, Semnan, Iran \\ ${ }^{2}$ Research Group of Nonlinear Analysis and Applications (RGNAA), Semnan, Iran \\ ${ }^{3}$ Centre of Excellence in Nonlinear Analysis and Applications (CENAA), Semnan University, \\ Semnan, Iran \\ Correspondence should be addressed to M. Eshaghi Gordji, madjid.eshaghi@gmail.com \\ Received 11 November 2010; Revised 11 January 2011; Accepted 19 January 2011 \\ Academic Editor: Yuming Shi
}

Copyright (C) 2011 M. Eshaghi Gordji and Z. Alizadeh. This is an open access article distributed under the Creative Commons Attribution License, which permits unrestricted use, distribution, and reproduction in any medium, provided the original work is properly cited.

Using fixed point methods, we prove the superstability and generalized Hyers-Ulam stability of ring homomorphisms on non-Archimedean Banach algebras. Moreover, we investigate the superstability of ring homomorphisms in non-Archimedean Banach algebras associated with the Jensen functional equation.

\section{Introduction and Preliminaries}

In 1897, Hensel [1] has introduced a normed space which does not have the Archimedean property.

During the last three decades theory of non-Archimedean spaces has gained the interest of physicists for their research in particular in problems coming from quantum physics, $p$-adic strings, and superstrings [2]. Although many results in the classical normed space theory have a non-Archimedean counterpart, their proofs are essentially different and require an entirely new kind of intuition [3-10].

Let $\mathbb{K}$ be a field. A non-Archimedean absolute value on $\mathbb{K}$ is a function $|\cdot|: \mathbb{K} \rightarrow \mathbb{R}$ such that for any $a, b \in \mathbb{K}$ we have

(i) $|a| \geq 0$ and equality holds if and only if $a=0$,

(ii) $|a b|=|a||b|$,

(iii) $|a+b| \leq \max \{|a|,|b|\}$. 
Condition (iii) is called the strict triangle inequality. By (ii), we have $|1|=|-1|=1$. Thus, by induction, it follows from (iii) that $|n| \leq 1$ for each integer $n$. We always assume in addition that $|\cdot|$ is non trivial, that is, that there is an $a_{0} \in \mathbb{K}$ such that $\left|a_{0}\right| \notin\{0,1\}$.

Let $X$ be a linear space over a scalar field $\mathbb{K}$ with a non-Archimedean nontrivial valuation $|\cdot|$. A function $\|\cdot\|: X \rightarrow \mathbb{R}$ is a non-Archimedean norm (valuation) if it satisfies the following conditions:

(NA1) $\|x\|=0$ if and only if $x=0$;

(NA2) $\|r x\|=|r|\|x\|$ for all $r \in \mathbb{K}$ and $x \in X$;

(NA3) the strong triangle inequality (ultrametric); namely,

$$
\|x+y\| \leq \max \{\|x\|,\|y\|\} \quad(x, y \in X) .
$$

Then $(X,\|\cdot\|)$ is called a non-Archimedean space.

It follows from $(N A 3)$ that

$$
\left\|x_{m}-x_{l}\right\| \leq \max \left\{\left\|x_{\jmath+1}-x_{\jmath}\right\|: l \leq \jmath \leq m-1\right\} \quad(m>l)
$$

and therefore a sequence $\left\{x_{m}\right\}$ is Cauchy in $X$ if and only if $\left\{x_{m+1}-x_{m}\right\}$ converges to zero in a non-Archimedean space. By a complete non-Archimedean space we mean one in which every Cauchy sequence is convergent. A non-Archimedean Banach algebra is a complete non-Archimedean algebra $\mathcal{A}$ which satisfies $\|a b\| \leq\|a\|\|b\|$ for all $a, b \in \mathcal{A}$. For more detailed definitions of non-Archimedean Banach algebras, we can refer to [11].

The first stability problem concerning group homomorphisms was raised by S. M. Ulam [12] in 1940 and affirmatively solved by D. H. Hyers [13]. Perhaps T. Aoki was the first author who has generalized the theorem of Hyers (see [14]).

T. M. Rassias [15] provided a generalization of Hyers' theorem which allows the Cauchy difference to be unbounded.

Theorem 1.1 (T. M. Rassias). Let $f: E \rightarrow E^{\prime}$ be a mapping from a normed vector space $E$ into a Banach space $E^{\prime}$ subject to the inequality

$$
\|f(x+y)-f(x)-f(y)\| \leq \epsilon\left(\|x\|^{p}+\|y\|^{p}\right)
$$

for all $x, y \in E$, where $\epsilon$ and $p$ are constants with $\epsilon>0$ and $p<1$. Then the limit

$$
L(x)=\lim _{n \rightarrow \infty} \frac{f\left(2^{n} x\right)}{2^{n}}
$$

exists for all $x \in E$ and $L: E \rightarrow E^{\prime}$ is the unique additive mapping which satisfies

$$
\|f(x)-L(x)\| \leq \frac{2 \epsilon}{2-2^{p}}\|x\|^{p}
$$

for all $x \in E$. Also, if for each $x \in E$ the mapping $f(t x)$ is continuous in $t \in \mathbb{R}$, then $L$ is $\mathbb{R}$-linear. 
Moreover, D. G. Bourgin [16] and Găvruţa [17] have considered the stability problem with unbounded Cauchy differences (see also [18-23]).

On the other hand, J. M. Rassias [24-29] considered the Cauchy difference controlled by a product of different powers of norm. However, there was a singular case; for this singularity a counterexample was given by Găvruţa [30].

Theorem 1.2 (J. M. Rassias [24]). Let X be a real normed linear space and $Y$ a real complete normed linear space. Assume that $f: X \rightarrow Y$ is an approximately additive mapping for which there exist constants $\theta \geq 0$ and $p, q \in \mathbb{R}$ such that $r=p+q \neq 1$ and $f$ satisfies the inequality

$$
\|f(x+y)-f(x)-f(y)\| \leq \theta\|x\|^{p}\|y\|^{q}
$$

for all $x, y \in X$. Then there exists a unique additive mapping $L: X \rightarrow Y$ satisfying

$$
\|f(x)-L(x)\| \leq \frac{\theta}{\left|2^{r}-2\right|}\|x\|^{r}
$$

for all $x \in X$. If, in addition, $f: X \rightarrow Y$ is a mapping such that the transformation $t \mapsto f(t x)$ is continuous in $t \in \mathbb{R}$ for each fixed $x \in X$, then $L$ is an $\mathbb{R}$-linear mapping.

Bourgin $[16,31]$ is the first mathematician dealing with stability of (ring) homomorphism $f(x y)=f(x) f(y)$. The topic of approximate homomorphisms was studied by a number of mathematicians, see [32-37] and references therein. A function $f: A \rightarrow A$ is a ring homomorphism or additive homomorphism if $f$ is an additive function satisfying

$$
f(x y)=f(x) f(y)
$$

for all $x, y \in A$.

Now we will state the following notion of fixed point theory. For the proof, refer to [38], see also [39, chapter 5]. For an extensive theory of fixed point theorems and other nonlinear methods, the reader is referred to [40,41]. In 2003, Radu [42] proposed a new method for obtaining the existence of exact solutions and error estimations, based on the fixed point alternative (see also [43-45]).

Let $(X, d)$ be a generalized metric space. An operator $T: X \rightarrow X$ satisfies a Lipschitz condition with Lipschitz constant $L$ if there exists a constant $L \geq 0$ such that $d(T x, T y) \leq$ $L d(x, y)$ for all $x, y \in X$. If the Lipschitz constant $L$ is less than 1 , then the operator $T$ is called a strictly contractive operator. Note that the distinction between the generalized metric and the usual metric is that the range of the former is permitted to include the infinity. We recall the following theorem by Margolis and Diaz.

Theorem 1.3 (cf. $[38,42]$ ). Suppose that one is given a complete generalized metric space $(\Omega, d)$ and a strictly contractive mapping $T: \Omega \rightarrow \Omega$ with Lipschitz constant L. Then for each given $x \in \Omega$, either

$$
d\left(T^{m} x, T^{m+1} x\right)=\infty \quad \forall m \geq 0
$$

or there exists a natural number $m_{0}$ such that 
(i) $d\left(T^{m} x, T^{m+1} x\right)<\infty$ for all $m \geq m_{0}$,

(ii) the sequence $\left\{T^{m} x\right\}$ is convergent to a fixed point $y^{*}$ of $T$;

(iii) $y^{*}$ is the unique fixed point of $T$ in $\Lambda=\left\{y \in \Omega: d\left(T^{m_{0}} x, y\right)<\infty\right\}$;

(iv) $d\left(y, y^{*}\right) \leq(1 /(1-L)) d(y, T y)$ for all $y \in \Lambda$.

Recently, the first author of the present paper [4] established the stability of ring homomorphisms on non-Archimedean Banach algebras. In this paper, using fixed point methods, we prove the generalized Hyers-Ulam stability of ring homomorphisms on non-Archimedean Banach algebras. Moreover, we investigate the superstability of ring homomorphisms on non-Archimedean Banach algebras associated with the Jensen functional equation.

\section{Approximation of Ring Homomorphisms in Non-Archimedean Banach Algebras}

Throughout this section we suppose that $A, B$ are two non-Archimedean Banach algebras. For convenience, we use the following abbreviation for a given function $f: A \rightarrow B$ :

$$
\Delta f(x, y)=f(x+y)-f(x)-f(y)
$$

for all $x, y \in A$.

Theorem 2.1. Let $f: A \rightarrow B$ be a function for which there exist functions $\varphi, \psi: A \times A \rightarrow[0, \infty)$ such that

$$
\begin{gathered}
\|\Delta f(x, y)\| \leq \varphi(x, y), \\
\|f(x y)-f(x) f(y)\| \leq \psi(x, y)
\end{gathered}
$$

for all $x, y \in A$. If there exists a constant $0<L<1$ such that

$$
\begin{gathered}
\varphi(2 x, 2 y) \leq|2| L \varphi(x, y) \\
\psi(2 x, 2 y) \leq|2|^{2} L \psi(x, y)
\end{gathered}
$$

for all $x, y \in A$, then there exists a unique ring homomorphism $H: A \rightarrow B$ such that

$$
\|f(x)-H(x)\| \leq \frac{1}{|2|(1-L)} \varphi(x, x),
$$

for all $x \in A$. 
Proof. It follows from (2.4) that

$$
\begin{aligned}
& \lim _{n \rightarrow \infty} \frac{1}{|2|^{n}} \varphi\left(2^{n} x, 2^{n} y\right)=0 \\
& \lim _{n \rightarrow \infty} \frac{1}{|2|^{2 n}} \psi\left(2^{n} x, 2^{n} y\right)=0
\end{aligned}
$$

for all $x, y \in X$. By $(2.6), \lim _{n \rightarrow \infty}\left(1 /|2|^{n}\right) \varphi(0,0)=0$. Hence, $\varphi(0,0)=0$. Letting $x=y=0$ in (2.2), we get $\|f(0)\| \leq \varphi(0,0)=0$. So $f(0)=0$.

Let us define $\Omega$ to be the set of all mappings $g: A \rightarrow B$ and introduce a generalized metric on $\Omega$ as follows:

$$
d(g, h)=\inf \{K \in(0, \infty):\|g(x)-h(x)\| \leq K \varphi(x, x), \forall x \in A\} .
$$

It is easy to show that $(\Omega, d)$ is a generalized complete metric space $[44,45]$.

Now we consider the function $T: \Omega \rightarrow \Omega$ defined by $T g(x)=(1 / 2) g(2 x)$ for all $x \in A$ and all $g \in \Omega$. Note that for all $g, h \in \Omega$,

$$
\begin{aligned}
d(g, h)<K & \Longrightarrow\|g(x)-h(x)\| \leq K \varphi(x, x), \quad \forall x \in A, \\
& \Longrightarrow\left\|\frac{1}{2} g(2 x)-\frac{1}{2} h(2 x)\right\| \leq \frac{1}{|2|} K \varphi(2 x, 2 x), \quad \forall x \in A, \\
& \Longrightarrow\left\|\frac{1}{2} g(2 x)-\frac{1}{2} h(2 x)\right\| \leq L K \varphi(x, x), \quad \forall x \in A, \\
& \Longrightarrow d(T g, T h) \leq L K .
\end{aligned}
$$

Hence, we see that

$$
d(T g, T h) \leq L d(g, h)
$$

for all $g, h \in \Omega$, that is, $T$ is a strictly self-function of $\Omega$ with the Lipschitz constant $L$.

Putting $y:=x$ in (2.2), we have

$$
\|f(2 x)-2 f(x)\| \leq \varphi(x, x)
$$

for all $x \in A$. So

$$
\left\|f(x)-\frac{1}{2} f(2 x)\right\| \leq \frac{1}{|2|} \varphi(x, x)
$$

for all $x \in A$, that is, $d(f, T f) \leq 1 /|2|<\infty$. 
Now, from the fixed point alternative, it follows that there exists a fixed point $H$ of $T$ in $\Omega$ such that

$$
H(x)=\lim _{n \rightarrow \infty} \frac{1}{2^{n}} f\left(2^{n} x\right)
$$

for all $x \in A$, since $\lim _{n \rightarrow \infty} d\left(T^{n} f, H\right)=0$.

On the other hand it follows from (2.2), (2.6), and (2.13) that

$$
\|\Delta H(x, y)\|=\lim _{n \rightarrow \infty} \frac{1}{|2|^{n}}\left\|\Delta f\left(2^{n} x, 2^{n} y\right)\right\| \leq \lim _{n \rightarrow \infty} \frac{1}{|2|^{n}} \varphi\left(2^{n} x, 2^{n} y\right)=0
$$

for all $x, y \in A$. So $\Delta H(x, y)=0$. This means that $H$ is additive. So it follows from the definition of $H,(2.3),(2.7)$, and (2.13) that

$$
\|H(x y)-H(x) H(y)\|=\lim _{n \rightarrow \infty} \frac{1}{|2|^{2 n}}\left\|f\left(2^{2 n} x y\right)-f\left(2^{n} x\right) f\left(2^{n} y\right)\right\| \leq \lim _{n \rightarrow \infty} \frac{1}{|2|^{2 n}} \psi\left(2^{2 n} x, 2^{2 n} y\right)=0
$$

for all $x, y \in A$. So $H(x y)=H(x) H(y)$. According to the fixed point alterative, since $H$ is the unique fixed point of $T$ in the set $\Lambda=\{g \in \Omega: d(f, g)<\infty\}, H$ is the unique function such that

$$
\|f(x)-H(x)\| \leq K \varphi(x, x)
$$

for all $x \in A$ and $K>0$. Again using the fixed point alternative, we get

$$
d(f, H) \leq \frac{1}{1-L} d(f, T f) \leq \frac{1}{|2|(1-L)}
$$

and so we conclude that

$$
\|f(x)-H(x)\| \leq \frac{1}{|2|(1-L)} \varphi(x, x)
$$

for all $x \in A$. This completes the proof.

Corollary 2.2. Let $\theta, p$, s be nonnegative real numbers with $p$, and $s>1$ and $2 s-2 p \geq 1$. Suppose that $f: A \rightarrow B$ is a function such that

$$
\begin{gathered}
\|\Delta f(x, y)\| \leq \theta\left(\|x\|^{p} \cdot\|y\|^{p}\right) \\
\|f(x y)-f(x) f(y)\| \leq \theta\left(\|x\|^{s} \cdot\|y\|^{s}\right)
\end{gathered}
$$


for all $x, y \in A$. Then there exists a unique ring homomorphism $H: A \rightarrow B$ satisfying

$$
\|f(x)-H(x)\| \leq \frac{\theta}{|2|-|2|^{2 p}}\|x\|^{2 p},
$$

for all $x \in A$.

Proof. The proof follows from Theorem 2.1 by taking

$$
\varphi(x, y):=\theta\left(\|x\|^{p} \cdot\|y\|^{p}\right), \quad \psi(x, y):=\theta\left(\|x\|^{s} \cdot\|y\|^{s}\right)
$$

for all $x, y \in A$. Then we can choose $L=|2|^{2 p-1}$ and we get the desired results.

Remark 2.3. Let $f: A \rightarrow B$ be a function for which there exist functions $\varphi, \psi: A \times A \rightarrow[0, \infty)$ satisfying (2.2) and (2.3). Let $0<L<1$ be a constant such that $\varphi(x / 2, y / 2) \leq(L /|2|) \varphi(x, y)$ for all $x, y \in A$. By a similar method to the proof of Theorem 2.1, one can show that there exists a unique ring homomorphism $H: A \rightarrow B$ satisfying

$$
\|f(x)-H(x)\| \leq \frac{L}{|2|(1-L)} \varphi(x, x) .
$$

For the case $\varphi(x, y):=\delta+\theta\left(\|x\|^{p} \cdot\|y\|^{p}\right.$ ) (where $\theta, \delta$ are nonnegative real numbers and $0<2 p<1$ ), there exists a unique ring homomorphism $H: A \rightarrow B$ satisfying

$$
\|f(x)-H(x)\| \leq \frac{\delta}{|2|^{2 p}-|2|}+\frac{\theta}{|2|^{2 p}-|2|}\|x\|^{2 p}
$$

for all $x \in A$.

In the following we establish the superstability of ring homomorphisms on nonArchimedean Banach algebras associated with the Jensen functional equation $f((x+y) / 2)=$ $(f(x)+f(y)) / 2$.

Theorem 2.4. Suppose there exist functions $\varphi, \psi: A \times A \rightarrow[0, \infty)$ such that there exists a constant $0<L<1$ such that

$$
\begin{gathered}
\varphi(0,2 y) \leq|2| L \varphi(0, y), \\
\psi(2 x, 2 y) \leq|2|^{2} L \psi(x, y)
\end{gathered}
$$

for all $x, y \in A$. Moreover, assume that $f: A \rightarrow B$ is a function such that

$$
\begin{gathered}
\left\|f\left(\frac{x+y}{2}\right)-\frac{f(x)+f(y)}{2}\right\| \leq \varphi(0, y), \\
\|f(x y)-f(x) f(y)\| \leq \psi(x, y)
\end{gathered}
$$

for all $x, y \in A$. Then $f$ is a ring homomorphism. 
Proof. Let us define $\Omega, d$ and $T: \Omega \rightarrow \Omega$ by the same definitions as in the proof of Theorem 2.1. By the same reasoning as in the proof of Theorem 2.1, one can show that $T$ has a (unique) fixed point $H$ in $\Omega$ such that

$$
H(x)=\lim _{n \rightarrow \infty} \frac{1}{2^{n}} f\left(2^{n} x\right)
$$

for all $x \in A$ and $H: A \rightarrow B$ is a ring homomorphism. On the other hand by the same reasoning as in the proof of Theorem 2.1, we can prove that $\varphi(0,0)=0$ and $f(0)=0$. Also, letting $y=0$ in (2.25), we get $f(x / 2)=f(x) / 2$ for all $x \in A$ (see $[24,25])$. So, by uniqueness property of $H$, we have $H=f$. It follows that $f$ is a ring homomorphism.

Corollary 2.5. Let $\theta, p$, and s be nonnegative real numbers with $p, s>1$. Suppose that $f: A \rightarrow B$ is a function such that

$$
\left\|f\left(\frac{x+y}{2}\right)-\frac{f(x)+f(y)}{2}\right\| \leq \theta\|y\|^{p}, \quad\|f(x y)-f(x) f(y)\| \leq \theta\left(\|x\|^{s} \cdot\|y\|^{s}\right)
$$

for all $x, y \in A$. Then $f$ is a ring homomorphism.

\section{References}

[1] K. Hensel, "Über eine neue begründung der theorie der algebraischen zahlen," in Jahresbericht der Deutschen Mathematiker-Vereinigung, vol. 6, pp. 83-88, 1897.

[2] A. Khrennikov, Non-Archimedean Analysis: Quantum Paradoxes, Dynamical Systems and Biological Models, vol. 427 of Mathematics and Its Applications, Kluwer Academic Publishers, Dordrecht, The Netherlands, 1997.

[3] L. M. Arriola and W. A. Beyer, "Stability of the Cauchy functional equation over $p$-adic fields," Real Analysis Exchange, vol. 31, no. 1, pp. 125-132, 2006.

[4] M. Eshaghi Gordji, "Nearly ring homomorphisms and nearly ring derivations on non-Archimedean banach algebras," Abstract and Applied Analysis, vol. 2010, Article ID 393247, 12 pages, 2010.

[5] M. Eshaghi Gordji and M. B. Savadkouhi, "Stability of cubic and quartic functional equations in nonArchimedean spaces," Acta Applicandae Mathematicae, vol. 110, no. 3, pp. 1321-1329, 2010.

[6] M. Eshaghi Gordji and M. B. Savadkouhi, "Stability of a mixed type cubic-quartic functional equation in non-Archimedean spaces," Applied Mathematics Letters, vol. 23, no. 10, pp. 1198-1202, 2010.

[7] M. Eshaghi Gordji, H. Khodaei, and R. Khodabakhsh, "General quartic-cubic-quadratic functional equation in non-Archimedean normed spaces," "Politehnica" University of Bucharest. Scientific Bulletin. Series A, vol. 72, no. 3, pp. 69-84, 2010.

[8] L. Narici and E. Beckenstein, "Strange terrain-non-Archimedean spaces," The American Mathematical Monthly, vol. 88, no. 9, pp. 667-676, 1981.

[9] C. Park, D. H. Boo, and T. M. Rassias, "Approximately addtive mappings over $p$-adic fields," Journal of Chungcheong Mathematical Society, vol. 21, pp. 1-14, 2008.

[10] V. S. Vladimirov, I. V. Volovich, and E. I. Zelenov, p-adic Analysis and Mathematical Physics, World Scientific, Singapore, 1994.

[11] N. Shilkret, Non-Archimedian Banach algebras, Ph.D. thesis, Polytechnic University, 1968, ProQuest LLC.

[12] S. M. Ulam, A Collection of the Mathematical Problems, Interscience Publishers, New York, 1940.

[13] D. H. Hyers, "On the stability of the linear functional equation," Proceedings of the National Academy of Sciences of the United States of America, vol. 27, pp. 222-224, 1941.

[14] T. Aoki, "On the stability of the linear transformation in Banach spaces," Journal of the Mathematical Society of Japan, vol. 2, pp. 64-66, 1950. 
[15] T. M. Rassias, "On the stability of the linear mapping in Banach spaces," Proceedings of the American Mathematical Society, vol. 72, no. 2, pp. 297-300, 1978.

[16] D. G. Bourgin, "Classes of transformations and bordering transformations," Bulletin of the American Mathematical Society, vol. 57, pp. 223-237, 1951.

[17] P. Găvruţa, "A generalization of the Hyers-Ulam-Rassias stability of approximately additive mappings," Journal of Mathematical Analysis and Applications, vol. 184, no. 3, pp. 431-436, 1994.

[18] R. Badora, "On approximate derivations," Mathematical Inequalities \& Applications, vol. 9, no. 1, pp. 167-173, 2006.

[19] R. Farokhzad and S. A. R. Hosseinioun, "Perturbations of Jordan higher derivations in Banach ternary algebras: an alternative fixed point approach," International Journal of Nonlinear Analysis and Applications, vol. 1, no. 1, pp. 42-53, 2010.

[20] M. Eshaghi Gordji, M. B. Savadkouhi, and M. Bidkham, "Stability of a mixed type additive and quadratic functional equation in non-Archimedean spaces," Journal of Computational Analysis and Applications, vol. 12, no. 2, pp. 454-462, 2010.

[21] S.-M. Jung, "On the Hyers-Ulam-Rassias stability of approximately additive mappings," Journal of Mathematical Analysis and Applications, vol. 204, no. 1, pp. 221-226, 1996.

[22] C. Park and M. E. Gordji, "Comment on approximate ternary Jordan derivations on Banach ternary algebras," Journal of Mathematical Physics, vol. 51, 7 pages, 2010, B. Savadkouhi, Journal of Mathematical Physics, vol. 50, article 042303, 2010.

[23] C. Park and A. Najati, "Generalized additive functional inequalities in Banach algebras," International Journal of Nonlinear Analysis and Applications, vol. 1, no. 2, pp. 54-62, 2010.

[24] J. M. Rassias, “On approximation of approximately linear mappings by linear mappings," Journal of Functional Analysis, vol. 46, no. 1, pp. 126-130, 1982.

[25] J. M. Rassias, "On approximation of approximately linear mappings by linear mappings," Bulletin des Sciences Mathématiques, vol. 108, no. 4, pp. 445-446, 1984.

[26] J. M. Rassias, "Solution of a problem of Ulam," Journal of Approximation Theory, vol. 57, no. 3, pp. 268-273, 1989.

[27] J. M. Rassias, "On the stability of the Euler-Lagrange functional equation," Chinese Journal of Mathematics, vol. 20, no. 2, pp. 185-190, 1992.

[28] J. M. Rassias, "Solution of a stability problem of Ulam,” Discussiones Mathematicae, vol. 12, pp. 95-103, 1992.

[29] J. M. Rassias, "Complete solution of the multi-dimensional problem of Ulam," Discussiones Mathematicae, vol. 14, pp. 101-107, 1994.

[30] P. Găvruta, "An answer to a question of John. M. Rassias concerning the stability of Cauchy equation," in Advances in Equations and Inequalities, Hardronic MaT. Series, pp. 67-71, 1999.

[31] D. G. Bourgin, "Approximately isometric and multiplicative transformations on continuous function rings," Duke Mathematical Journal, vol. 16, pp. 385-397, 1949.

[32] R. Badora, "On approximate ring homomorphisms," Journal of Mathematical Analysis and Applications, vol. 276, no. 2, pp. 589-597, 2002.

[33] J. Baker, J. Lawrence, and F. Zorzitto, "The stability of the equation $f(x+y)=f(x) f(y)$," Proceedings of the American Mathematical Society, vol. 74, no. 2, pp. 242-246, 1979.

[34] M. Eshaghi Gordji, T. Karimi, and S. Kaboli Gharetapeh, "Approximately $n$-Jordan homomorphisms on Banach algebras," Journal of Inequalities and Applications, vol. 2009, Article ID 870843, 8 pages, 2009.

[35] M. Eshaghi Gordji and A. Najati, "Approximately J*-homomorphisms: a fixed point approach," Journal of Geometry and Physics, vol. 60, no. 5, pp. 809-814, 2010.

[36] C.-G. Park, "Homomorphisms between Lie $J C^{*}$-algebras and Cauchy-Rassias stability of Lie JC*algebra derivations," Journal of Lie Theory, vol. 15, no. 2, pp. 393-414, 2005.

[37] C.-G. Park, "Lie *-homomorphisms between Lie $C^{*}$-algebras and Lie $*$-derivations on Lie $C^{*}$ algebras," Journal of Mathematical Analysis and Applications, vol. 293, no. 2, pp. 419-434, 2004.

[38] J. B. Diaz and B. Margolis, "A fixed point theorem of the alternative, for contractions on a generalized complete metric space," Bulletin of the American Mathematical Society, vol. 74, pp. 305-309, 1968.

[39] I. A. Rus, Principles and Applications of Fixed Point Theory, Dacia, Cluj-Napoca, Romania, 1979.

[40] D. H. Hyers, G. Isac, and T. M. Rassias, Stability of Functional Equations in Several Variables, Birkhäuser Boston, Boston, Mass, USA, 1998.

[41] E. Zeidler, Nonlinear Functional Analysis and Its Applications. I, Springer, New York, NY, USA, 1986.

[42] V. Radu, "The fixed point alternative and the stability of functional equations," Fixed Point Theory, vol. 4, no. 1, pp. 91-96, 2003. 
[43] L. Cădariu and V. Radu, "Fixed points and the stability of Jensen's functional equation," Journal of Inequalities in Pure and Applied Mathematics, vol. 4, no. 1, article 4, p. 7, 2003.

[44] L. Cădariu and V. Radu, "On the stability of the Cauchy functional equation: a fixed point approach," in Iteration Theory, vol. 346, pp. 43-52, Karl-Franzens-Universitaet Graz, 2004.

[45] L. Cădariu and V. Radu, "Fixed point methods for the generalized stability of functional equations in a single variable," Fixed Point Theory and Applications, vol. 2008, Article ID 749392, 15 pages, 2008. 


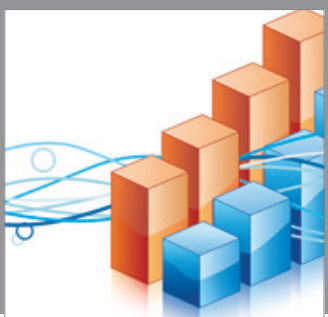

Advances in

Operations Research

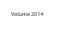

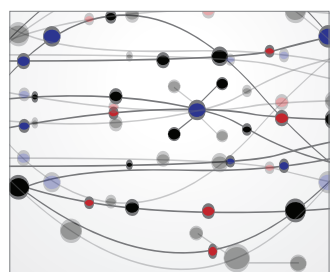

\section{The Scientific} World Journal
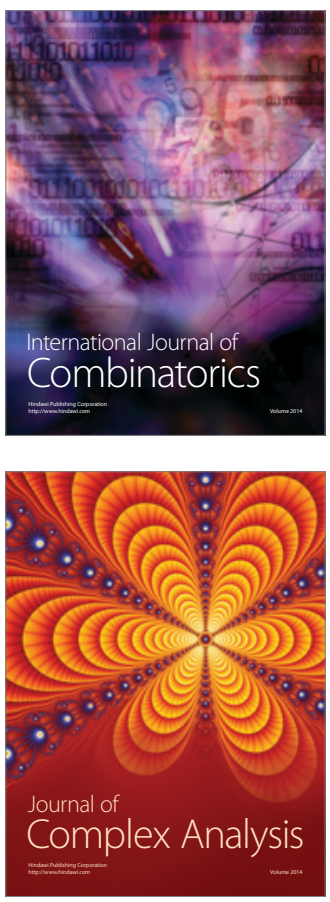

International Journal of

Mathematics and

Mathematical

Sciences
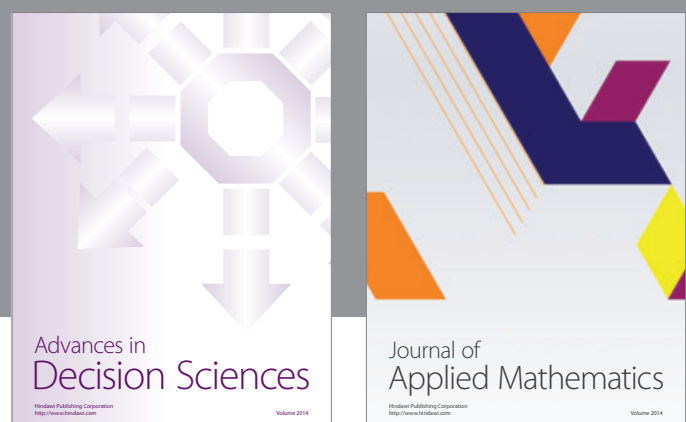

Journal of

Applied Mathematics
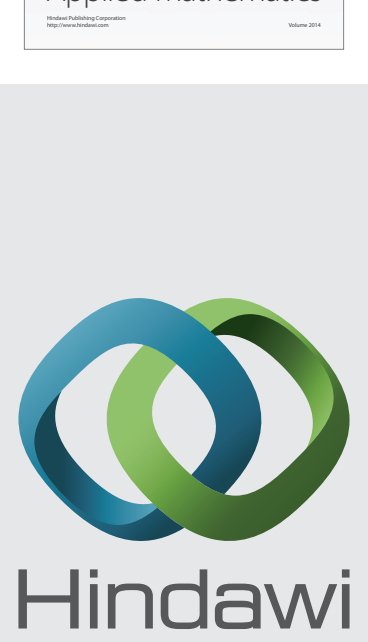

Submit your manuscripts at http://www.hindawi.com
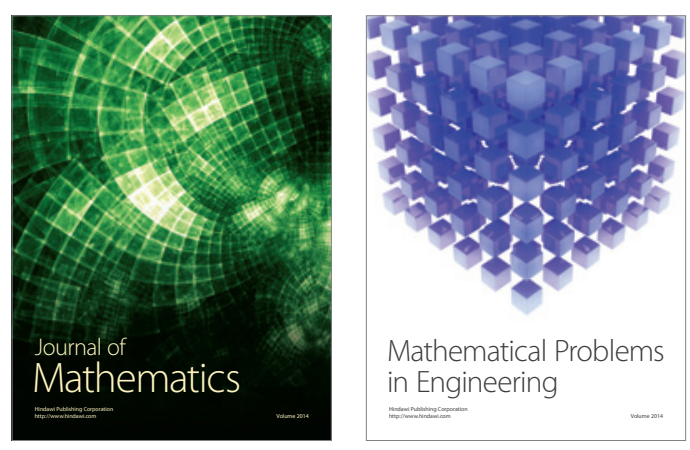

Mathematical Problems in Engineering
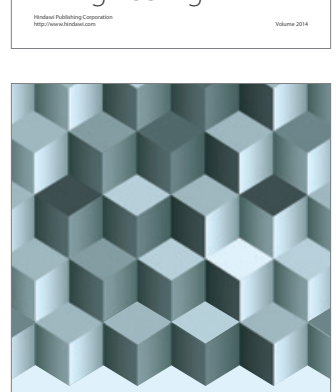

Journal of

Function Spaces
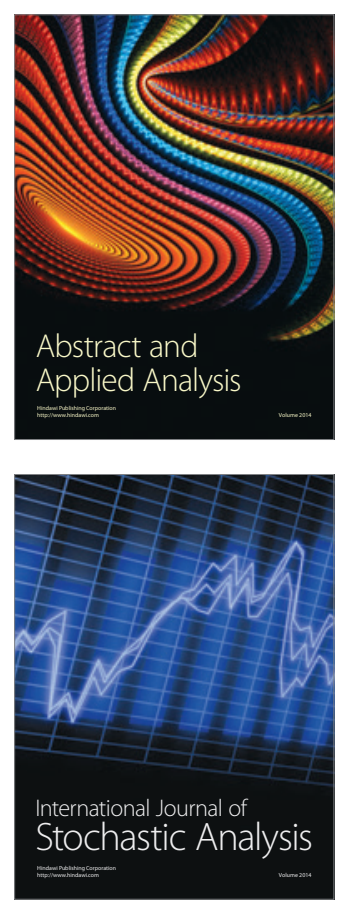

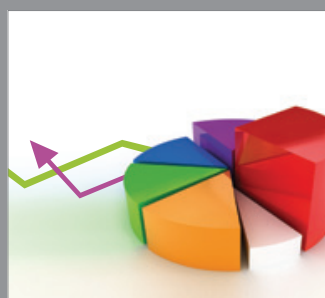

ournal of

Probability and Statistics

Promensencen
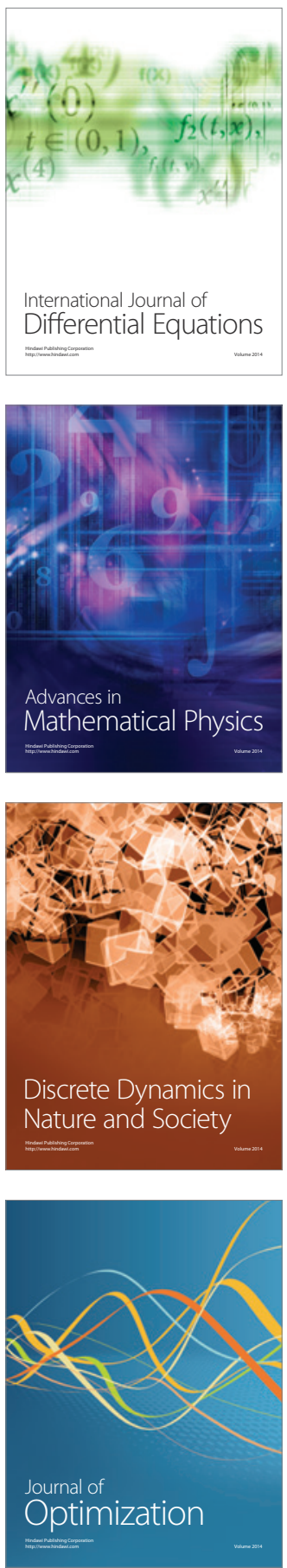